\title{
Severe hyponatremia, hypertension, acute deterioration of renal function in a young female with lupus nephritis
}

\author{
Ioana Saulescul, ${ }^{1,2}$, Sanziana Daia-Iliescu, ${ }^{1,2}$, Cosmin Laurentiu Constantinescu, ${ }^{1,2}$, \\ Andreea Borangiu ${ }^{1,2}$, Casandra Buzatu', Ruxandra Ionescu ${ }^{1,2}$, Daniela Opris-Belinski ${ }^{1,2}$ \\ ${ }^{1}$ Department of Internal Medicine and Rheumatology, "Sf. Maria" Clinical Hospital, Bucharest, Romania \\ 2"Carol Davila"University of Medicine and Pharmacy, Bucharest
}

\begin{abstract}
Lupus nephritis is one of the most important organ involvement in patients diagnosed with Systemic Lupus Erythematosus (SLE). It requires aggressive and prolonged immunosuppression in order to induce and maintain remission. There is always a risk of adverse events.

It is presented the case of a young woman diagnosed with SLE and active lupus nephritis with acute renal failure, who developed persistent hyponatremia after immunosuppression with Cyclophosphamide was started. The case emphases both the need for synthetic immunosuppression as well as the adverse events that may occur during treatment.

Close monitoring of the clinical and biological parameters during immunosuppressive therapy is essential as even one minimally modified parameter may prove important in the evolution of the case. Measures to prevent one side effect do not prevent the developing of other.
\end{abstract}

Keywords: lupus nephritis, SIADH, cyclophosphamide

\section{INTRODUCTION}

In complex systemic diseases like systemic lupus erythematosus (SLE), disease flares are interspersed with episodes of remission. Complications may occur due to the disease itself but also due to treatment so close monitoring of the patient's condition is necessary. The acknowledgement about existence of continuum accumulation of damage improved a lot the survival for these patients. When treatment is prescribed, it is important to prevent or promptly treat any side effect of drug therapy or any disease complication (1-3).

\section{CASE REPORT}

We are presenting the case of a 37-year-old woman admitted in the Department of Internal Medicine and Rheumatology for fatigue, lower extremity edema, high blood pressure, malar rush. The patient is known with systemic lupus erythematosus for 9 years with organ involvement (cutaneous, articularin year 2008, serositis - in year 2010, hematological - in year 2015). One year before the present episode, she developed lupus nephritis (most probably diffuse prolipherative glomerulonephritis) that required immunosuppression with cyclophosphamide according to 2012 recommendation for lupus nephritis. Induction therapy with high dose glucocorticoids and cyclophosphamide was followed by maintenance with mycophenolate mofetil (MMF) - discontinued by the patient after three months.

The present clinical examination revealed general malaise, excessive fatigue, malar rush, periocular and lower extremity edema with high blood pressure (180/100 $\mathrm{mmhg}$ ), and oligoanuria. Laboratory testing showed blood urea nitrogen level of $81 \mathrm{mg} / \mathrm{dl}$, creatinine $3,5 \mathrm{mg} / \mathrm{dl}-\mathrm{GFR}=20 \mathrm{ml} / \mathrm{min}$, serum proteins $5,8 \mathrm{~g} / \mathrm{dl}$, daily protein loss $2,3 \mathrm{~g}$ and active uri- 
nary sediment with red blood cells and cellular casts. Her complement (C3) levels were low, and also mild electrolyte imbalance K 5,8 mmol/1, Na $135 \mathrm{mmol} / \mathrm{l}$ was noticed at admittance. Antibody testing was positive for anti dsDNA, anti Sm, anti Ro and anti La and negative for circulating lupus anticoagulants. The echography showed presence of minimum pericarditis and normal kidney dimensions.

The patient has active LES, nephritic syndrome with hypertension and acute deterioration of renal function. His history and clinical, laboratory assessments suggest a prolipherative lupus nephritis with poor prognostic factor - acute renal failure. Induction immunosuppression was started according to the 2012 recommendations for management of lupus nephritis: cyclophosphamide $10 \mathrm{mg} / \mathrm{kg}(\mathrm{NIH})$ and $0,5 \mathrm{~g}$ /day of iv methylprednisolone for 4 days followed by oral prednisolone.

Four days later, the patient developed general weakness, nausea and aggravating edema of the lower limbs. The lab results showed an electrolyte imbalance with an alarming raise in her serum potassium concentration $(6,8 \mathrm{mmol} / \mathrm{l})$ and low levels of serum natrium $(125 \mathrm{mmol} / \mathrm{l})$. Supportive care was added with isotonic saline solutions and glucose, but the persistent hyperkalemia represented an indication for hemodialysis. After hemodialysis, the renal function was improved with mildly elevated creatinine and blood urea nitrogen level as well as normal potassium concentration. In spite of normal fluid balance, a persistent hyponatremia $(122 \mathrm{mmol} / \mathrm{l})$ is observed. In order to correct the hyponatremia intravenous isotonic saline solutions were administered along with loop diuretics still with no response.

Underlying causes of resistant hyponatremia have been taken into account. Additional lab results showed a normal range of plasmatic volume (due to correctly performed hemodialysis), hypo-osmolarity $(270 \mathrm{mOsm} / \mathrm{L})$ with normal urinary density and normal sodium excretion.

In the absence of structural brain lesions (clear head CT), no evidence for heart or liver failure (echocardiography, normal liver function), no hypothyroidism (TSH within the normal range), and adrenal insufficiency highly improbable as the patient was receiving cortisone treatment, the hyponatremia may be secondary to inappropriate secretion of antidiuretic hormone.

The patient fulfills the Bartter-Schwartz criteria for the diagnosis of inappropriate secretion of antidiuretic hormone syndrome (SIADH). Fluid restric- tion (less than $500 \mathrm{ml} /$ day oral intake and intravenous solutions) in addition to the loop diuretics that the patient already received led to rapid improvement of the clinical condition as well as a rise in her serum sodium level, another argument in favor of the SIADH diagnosis.

To sum up, a young patient with lupus nephritis and poor prognostic factors developed persistent, moderately-symptomatic hyponatremia. Hemodialysis was performed in order to resolve the acute renal failure and also partially corrected the electrolyte imbalance. Immunosuppression with cyclophosphamide, followed by mycophenolate mofetil was stared for the active lupus nephritis. Fluid restriction and loop diuretics resolved the persistent hyponatremia. The evolution was favorable with edema remission, weight loss, normal blood pressure, normal renal function and normal plasma electrolytes (Fig. 1).

\section{DISCUSSION}

Systemic lupus erythematosus (SLE) is a complex disease, with multiple organ involvement, but more over with variable severity. Disease evolution can go from periods of activity to periods of remission. It is also important to recognize complications that are possible due to SLE but also because of treatment. This is way acknowledgement about existence of continuum accumulation of damage improved a lot survival for these patients. Because of this variable course of the disease, effective management means close monitoring of the patients, control of the symptoms, prevent and prompt recognition of the relapses, but also prevent or treat side effects of drug therapy or disease complications (1-3).

Fig. 2 explains how should be our routine in front of a patient with SLE.

Treatment of SLE patients should be tailored according to clinical manifestation and severity, differentiating activity from irreversible damage. Reversible manifestations that are caused by the underlying inflammatory process account for active disease and for these type of manifestations patients need antimalarial drugs, anti-inflammatory drugs (non-steroidal or steroidal), immunosuppressive agents or biological treatment. Flare severity is the one that dictate intensity of treatment. In opposite, the irreversible organ manifestations reflect the damage and they should be treated specifically to organ involved (2-4). 

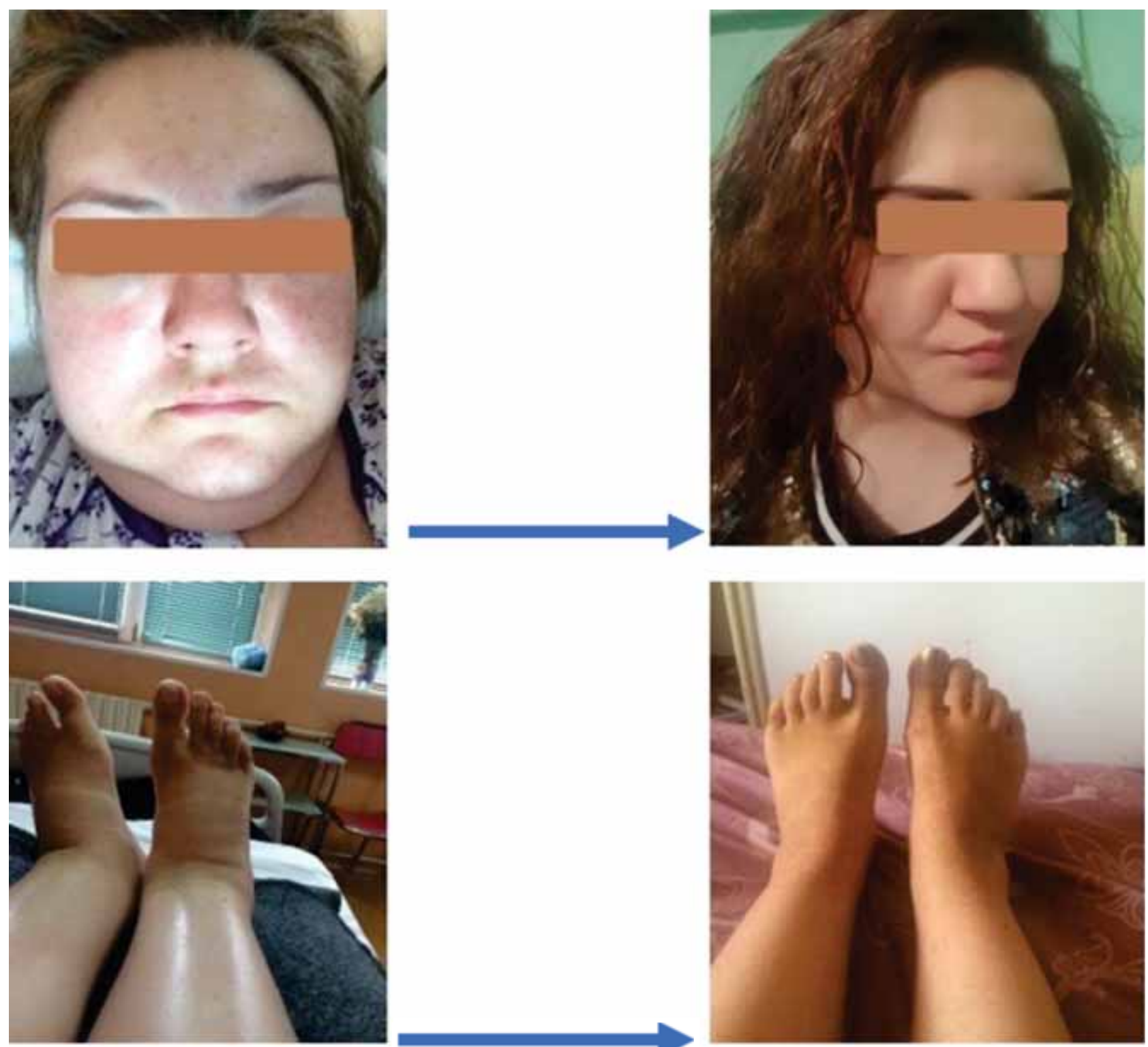

FIGURE 1. Clinical evolution over one month

In our case, the dominant active organ involvement was renal. She had a nephritic syndrome with hypertension and acute deterioration of renal function. We treated the patients according to 2012 recommendations for the management of lupus nephritis $(5,6)$. Clinical and laboratory assessment suggested presence of proliferative lupus nephritis (nephritic range proteinuria, active urinary sediment and poor prognostic factors because of the acute renal insufficiency). Immunosuppressive therapy for this type of suffering consists in two phases, both important for a successful outcome. These are induction and maintenance phase $(5,6)$.

Initial (induction) therapy has as a primary goal to achieve a renal response. Therefore, it involves the administration of potent anti-inflammatory and immunosuppressive drugs, meaning high-dose glucocorticoids and either cyclophosphamide $(\mathrm{Cyc})$ or mycophenolate mofetil (MMF). The duration of this period may vary between three months or six months $(5,6)$.

Because our patient had adverse prognostic factors, our option was to use cyclophosphamide at higher doses (10-15 mg/kg) for 6 months and three days course of intravenous methylprednisolone pulse therapy, followed by oral prednisone $0.5 \mathrm{mg} /$ $\mathrm{kg} /$ day for 4 weeks with intention to reduce the dose in the following months.

Supportive care was also added. Intravenous hydration with isotonic saline solution and glucose 5\% was administrated daily in order to prevent Cyc toxicity, but also to correct serum creatinine and elec- 


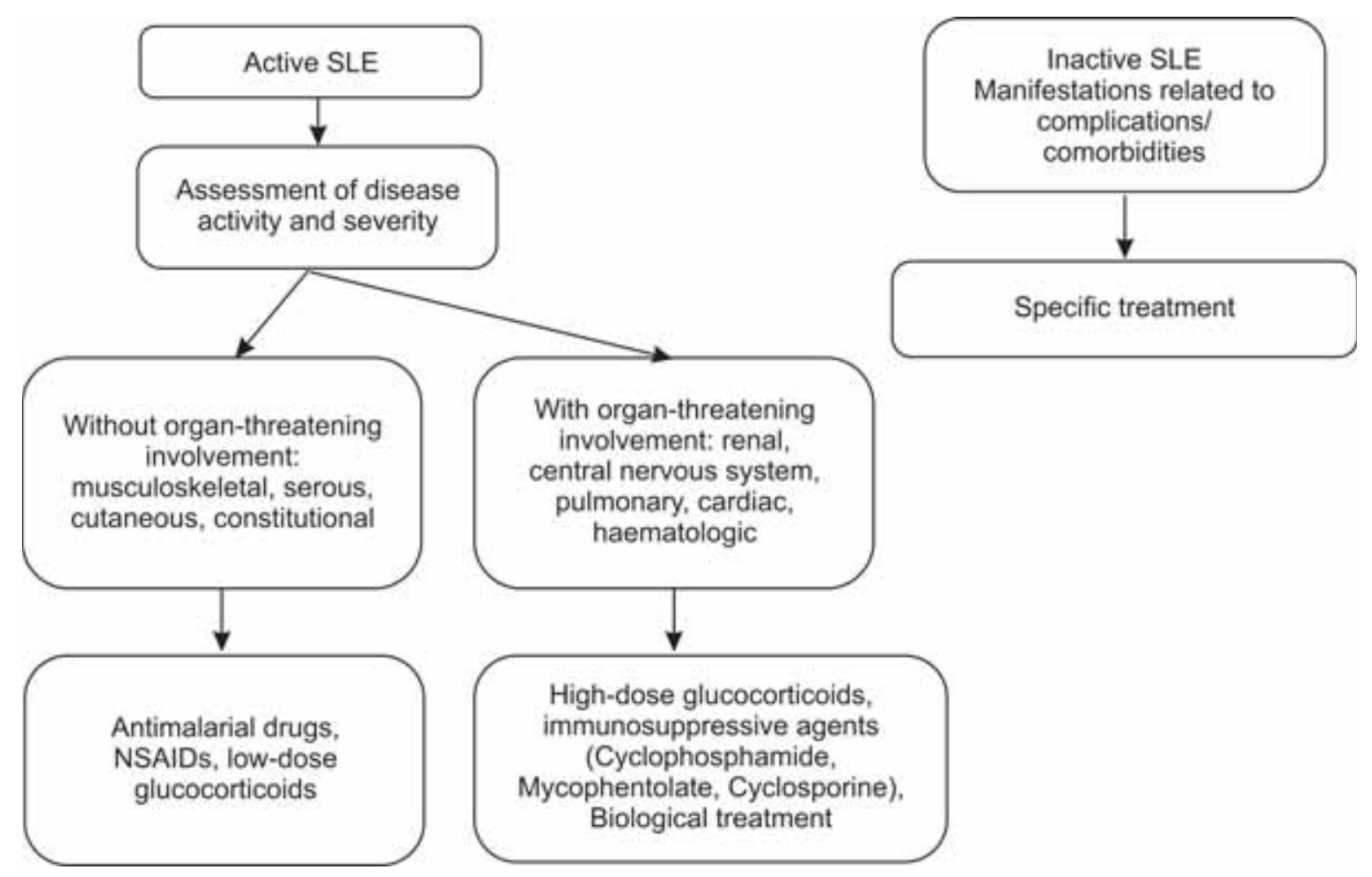

FIGURE 2. Proposed algorithm for evaluation and treatment of patients with SLE

trolytic imbalance: high potassium level with small decrease in serum sodium.

Although these measures, she continued to have persistent edema and increasing seric potassium, leading to hemodialysis. This maneuver corrected renal function and potassium level, but she developed nausea, with increasing edema and further reduction in serum sodium to an emergency level, with no response to administration of isotonic saline solution and loop diuretic. It was necessary to investigate abnormalities related to hyponatremia. In such case, the detection of the underlying cause of hyponatremia is very important for a proper treatment and should trigger evaluation of plasma osmolality, urine osmolality and urine sodium concentration (7).

The first step is to evaluate if you are in front of a true hyponatremia. In this case, plasma osmolality should be low. Pseudohyponatremia due to hyperglycemia, hyperlipidemia or hyperproteinemia, but also renal failure goes with normal or elevated plasma osmolality. In our case, this parameter was low, so the hyponatremia was true.

Next step must verify if water excretion is normal or impaired by urine osmolality. Urine osmolality higher than $100 \mathrm{msmol} / \mathrm{kg}$ - as in our case - is suggestive for true hyponatremia due to water impaired excretion.
Urine sodium in a spot urine specimen it was the next step in evaluation. Values higher than $40 \mathrm{~mol} / \mathrm{l}$ are found in inappropriate secretion of antidiuretic hormone syndrome (SIADH) and also in adrenal insufficiency, renal failure or due to excess of diuretics. None of the last three causes mentioned were compatible with our case, so our diagnosis was SIADH (7).

SIADH is a disorder of impaired water excretion triggered by the inability to suppress the secretion of antidiuretic hormone (ADH), leading to hyponatremia when water intake exceeds reduced urine output (8).

SIADH is defined by the Barttter-Schartz criteria that are summarized below:

- Hyponatremia with hypoosmolality $(<275$ $\operatorname{mosm} / \mathrm{kg}$ ),

- Normal renal sodium excretion (urine $\mathrm{Na}>20$ $\mathrm{mEq} / 1$ ),

- Urine osmolality $>100 \mathrm{mosm} / \mathrm{kg}$,

- Absence of clinical evidence of volume depletion,

- Absence of other causes of hyponatremia, such as renal failure, heart failure, endocrine pathology (thyroid, adrenal insufficiency) $(7,8)$. 
Major causes of SIADH are:

- pulmonary disorders: acute asthma, tuberculosis, carcinoma, pneumonia, acute respiratory failure, etc.,

- central nervous system (CNS) disorders: infection, stroke, tumors, trauma, psychosis, encephalopathy, SLE,

- cancer: pulmonary (small cell carcinoma), head and neck carcinoma, gastrointestinal carcinoma, genitourinary carcinoma

- drugs: cyclophosphamide, carbamazepine, non-steroidal anti-inflammatory drugs,

- following major surgery,

- idiopathic (7).

In the present case we took into consideration as possible cause for SIADH SLE itself or cyclophosphamide.

There is little information about SIADH related directly to SLE due to small size studies and reduced number of case reported. Most of the cases are associated with neurolupus, thrombotic or vasculitic lesions (9). More rare, SIADH was diagnosed in active SLE with other organ involvement than CNS and an intense immunology: high titer antinuclear antibody, double-strained antibody or presence of U1RNP antibody $(10,11)$.

A more probable etiology we think to be recent administration of Cyc. This complication was first described for high-doses intravenous Cyc (30-50 $\mathrm{mg} / \mathrm{kg}$ ) used to treat malignancy, but hyponatremia can occur also in lower doses $(10-15 \mathrm{mg} / \mathrm{kg})$ that are given for autoimmune disorder, lupus nephritis included (12-14). Chemotherapy induced nausea could also contribute to SIADH, because nausea stimulates the $\mathrm{ADH}$ release (15). Hyponatremia is further aggravated by the fluid overloaded that we use to prevent hemorrhagic cystitis.

This was probably our case, since hyponatremia in our patient further decrease after intravenous isotonic saline administration. In SIADH only water excretion is impaired, sodium handling is normal since is regulated by renin - angiotensin - aldosterone mechanism. If we give only isotonic saline solution with no further increase in solute intake there will be no correction of hyponatremia since urinary osmolality is fix.

Following measures are indicated to correct hyponatremia due to SIADH.

1. Fluid restriction: suggested goal intake is less than $800 \mathrm{ml} /$ day. The negative water balance will increase slowly serum sodium level.
2. Increasing solute intake (mostly sodium salts and urea): this will increase urinary excretion of solutes and concomitant water excretion. Oral salt intake should be around $9 \mathrm{~g} /$ day and associated with hyperproteic diet.

3. Loop diuretic: this impairs the renal responsiveness to ADH. Furosemide should be given daily, despite apparent contraindication because of hyponatremia.

4. Intravenous hypertonic saline solution, vasopressin receptor antagonists might be used in resistant cases or in major emergency hyponatremia (presence of symptoms like coma or convulsion, acute severe hyponatremia) (7).

After using first three measures, our patient recovered completely, fluid overload was corrected and sodium balance normalized.

Since our primary supposition is that SIADH was related to Cyc administration, we changed immunosuppressive regimen of induction therapy to mycophenolate mofetil and patient was discharged in good clinical state and normal laboratory parameters.

\section{CONCLUSIONS}

This case brings to light both the need for synthetic immunosuppression and the adverse events that can occur. Lupus nephritis is a severe organ involvement that needs prompt immunosuppressive treatment in order to obtain good outcomes.

Cyc remains an important tool for the treatment of these patients. One of more rare side effects is the inappropriate secretion of antidiuretic hormone syndrome, sometimes precipitated by iatrogenic fluid overload for the prevention of hemorrhagic cystitis. Measures to prevent one side effects do not prevent the developing of other. We underline the importance of close monitoring the clinical and biological parameters after administering immunosuppression, electrolytic balance including. Hyponatremia following Cyc administration requires prompt fluid restriction, loop diuretics and increase solute intake, mainly oral salt.

Conflict of interest: none declared Financial support: none declared 


\section{REFERENCES}

1. Bertsias G., Cervera R., Boumpas D.T. Systemic Lupus Erythematosus: pathogenesis and clinical features, in Eular Textbook on Rheumatic Diseases, 2012

2. Bertsias G. Therapeutic opportunities in SLE: state of the art and prospects for the new decade, Ann Rheum Dis 2010; 69: 16031611

3. Wallace D.J. Principles of therapy and local measures in DJ Wallace, BH Hahn, DuBois' Lupus Erythematosus and related Syndromes, edition an 8-a, Elsevier, 2012

4. Wallace D.J. et al. The management of lupus in DJ Wallace, BH Hahn, DuBois' Lupus Erythematosus and related Syndromes, edition a 8-a, Elsevier, 2012

5. Bertsias G., Tektonidou M. et al. Joint European League Against Rheumatism and European Renal Association recommendations for the management of adult lupus nephritis, Ann Rheum Dis, 2012; 201940

6. Hahn B. et al. American College of Rheumatology Recommendation for screening, treatment and management of lupus nephritis, Arthritis Care Res, 2012; 797-808

7. Elisaf M.S., Drosos A.A. Hyponatremia due to inappropriate secretion of antidiuretic hormone in a patient with systemic lupus erythematosus, Clinical Experimental Rheumatology, 1999, $17: 223-226$
8. Bartter F.C., Schwartz W.B. The syndrome of inappropriate secretion of antidiuretic hormone, AM J MED, 1967, 5: 790-806,

9. Kengne F.G. et al. Inappropriate secretion of $A D H$ and central diabetes insipidus are related to antiphospholipid antibodies in SLE, Nephron Dial Transplant, 2006

10. Agus B. et al. Inappropriate secretion of ADH in a patient with SLE, Arthritis Rheum, 1983

11. Trachtman H. et al. Abnormal antidiuretic hormone secretion in patients with SLE, Nephron, 1987

12. Bressler R.B., Huston D.P. Water intoxication following moderate dose intravenous Cyclophosphamide, Arch Inern Med, 1985

13. Salido M. et al. Water intoxication induced by low-dose Cyclophosphamide in two patients with systemic lupus erythematosus, Lupus, 2003,

14. Gilbar P.J. et al. Syndrome of inappropiate antidiuretic hormone secretion induced by a single dose of oral cyclophosphamide. Ann Pharmacotherap, 2012; 46( 9):e23;

15. Nausea and vasopressin: Pathophysiology and etiology of the syndrome of inappropriate antidiuretic hormone secretion, Lancet, 1991 